\title{
Germanica
}

\section{Palestrina(1917) de Hans Pfitzner : un opéra à la charnière de deux époques}

Hans Pfitzners Palestrina (1917): Oper einer Übergangszeit

Joëlle Stoupy

\section{OpenEdition}

\section{Journals}

Édition électronique

URL : http://journals.openedition.org/germanica/493

DOI : 10.4000/germanica.493

ISSN : 2107-0784

Éditeur

Université de Lille

Édition imprimée

Date de publication : 1 décembre 2007

Pagination : $35-43$

ISBN : 978-2-913857-20-9

ISSN : 0984-2632

Référence électronique

Joëlle Stoupy, «Palestrina(1917) de Hans Pfitzner : un opéra à la charnière de deux époques »,

Germanica [En ligne], 41 | 2007, mis en ligne le 01 décembre 2009, consulté le 06 octobre 2020. URL

http://journals.openedition.org/germanica/493 ; DOI : https://doi.org/10.4000/germanica.493

Ce document a été généré automatiquement le 6 octobre 2020.

(c) Tous droits réservés 


\title{
Palestrina(1917) de Hans Pfitzner : un opéra à la charnière de deux époques
}

\author{
Hans Pfitzners Palestrina (1917): Oper einer Übergangszeit
}

\author{
Joëlle Stoupy
}

1 Le 12 juin 1917, le public du Prinzregententheater à Munich acclame avec ferveur la nouvelle création du compositeur allemand Hans Pfitzner (1869-1949): la légende musicale en trois actes, Palestrina, qui se déroule dans l'Italie de la Renaissance, à l'époque du Concile de Trente. Le livret, rédigé par Pfitzner lui-même, est alors aussi apprécié que la musique. Le chef d'orchestre, Bruno Walter, parle de la première comme d'un des événements capitaux dans sa carrière et de l'œuvre de Pfitzner comme d'un «sommet dans la création musicale de notre époque "1. Thomas Mann, de son côté, connaît le texte avant d'avoir été spectateur de l'opéra et en fait l'éloge dans les Considérations d'un apolitique (1918), reconnaissant dans le livret de Palestrina l'antagonisme irréversible à ses yeux, à cette date, entre l'art et la politique.

2 Pfitzner lui-même considère Palestrina comme son œuvre maîtresse ${ }^{2}$. Le fait qu'il ait conçu et écrit le livret n'est pas étranger à cette appréciation. Une lettre qu'il adresse en 1929 au Théâtre National de Munich nous montre qu'il réclame avec fermeté pour lui, dans le cas de Palestrina, le titre de Dichterkomponist (compositeur-poète), ne se satisfaisant pas de celui de compositeur ${ }^{3}$, à ses yeux trop réducteur. En écrivant luimême le livret, Pfitzner a voulu être fidèle à sa première inspiration, décrire sous forme de triptyque le monde de l'art d'un côté, un monde de recueillement, d'intériorité et d'intemporalité, représenté par Palestrina dans l'acte I et III et, par opposition, le monde extérieur dans l'acte II, « avec son agitation bruyante et effrénée se déroulant dans la temporalité $»^{4}$; ainsi, aucune version composée par différents librettistes n'a pu le satisfaire et lui restituer cette vision première. Hésitant, ne se sentant pas en mesure d'être "poète $»^{5}$, Pfitzner franchira cependant le pas pour les besoins de l'œuvre et écrira le livret de 1910 au 7 août 1911 avant de composer la 
musique. "Je me souviens », dit Pfitzner, «ne pas m'être senti compositeur du tout pendant tout ce temps $»^{6}$.

3 À l'époque où il écrit Palestrina, Pfitzner réclame du livret d'opéra un niveau littéraire de premier rang - une exigence qu'il doit à Wagner. Il écrit à ce propos à Arthur Eloesser : « Tu connais bien mon point de vue sur l'opéra et sur la poésie en général ; et tu sais qu'il n'est pas frivole, et combien je méprise les gens qui, sans être poètes, se mettent à écrire eux-mêmes des 'textes' qu'ils considèrent comme des poésies ${ }^{7}$. Pfitzner refuse ainsi de voir le contenu idéel devenir accessoire. Comme le dit Thomas Mann admiratif, Pfitzner a voulu écrire avec Palestrina un "pur drame d'idées ${ }^{8}$. Pour Pfitzner, l'opéra doit alors, grâce entre autres à la qualité du livret, provoquer une émotion profonde et une élévation chez le spectateur, comme le réclamait déjà le drame classique.

Le personnage de Pierluigi Palestrina, compositeur italien du $\mathrm{xvI}^{\mathrm{e}}$ siècle, a retenu l'attention de Pfitzner de longues années avant la rédaction de l'opéra' ${ }^{9}$. L'œuvre de Pfitzner ne doit rien au drame Palestrina que Samuel Schier publie en 1825, pas plus qu'à l'opéra de Melchior Ernst Sachs qui porte le même titre. Pfitzner a reçu «la première et la plus forte impulsion pour la conception de [s]on œuvre $»^{10}$ de l'histoire de la musique d'August Wilhelm Ambros. Sans être un opéra historique, Palestrina doit aussi sa valeur au fait qu'il a fait l'objet de recherches historiques minutieuses, appliquées essentiellement à l'acte II consacré à la dernière session du Concile de Trente. Ces recherches assidues n'ont cependant pas empêché le compositeur de s'éloigner de ses sources historiques pour le bien de son idée première; le thème central de l'opéra étant la création d'un chef-d'œuvre musical, une messe, dans un monde étranger aux exigences de l'art. Cette idée première, Schopenhauer lui en donne les assises philosophiques. Dans l'œuvre Parerga et paralipomena que Pfitzner affectionne particulièrement et dont il met un passage en exergue dans le texte de Palestrina, Schopenhauer évoque deux mondes distincts, la vie intellectuelle planant «au-dessus de l'agitation du monde, de la vie réelle des peuples, celle qui est régie par la Volonté " ${ }^{11}$, de telle sorte qu'« à côté de l'histoire du monde, l'histoire de la philosophie, des sciences et des arts avance innocente et immaculée ».

On a appelé à juste titre Palestrina « une œuvre tardive dans l'histoire du Künstlerdrama allemand $»^{12}$, un genre qui, avec Pfitzner, touche à sa fin ${ }^{13}$. C'est Goethe qui le premier avec Torquato Tasso met un artiste au centre des débats de son drame, tandis qu'avant lui le personnage de l'artiste, quand il apparaissait, avait un rôle secondaire ${ }^{14}$. Au tournant $\mathrm{du} \mathrm{xIX}^{\mathrm{e}}$ au $\mathrm{xx}^{\mathrm{e}}$ siècle, l'antagonisme entre art et vie joue un rôle prépondérant dans la littérature et de nombreux auteurs tels que Hesse, Hauptmann, Hofmannsthal, Rilke, George ou Thomas Mann font de ce thème leur cheval de bataille, car la place de l'artiste dans la société de leur époque s'avère problématique. Pfitzner ressent luimême très tôt la difficulté de créer dans une époque toujours plus hostile à l'art tel qu'il le comprend : « Notre vie devient de plus en plus mécanique, de plus en plus prosaïque, hostile à toute intériorité $\aleph^{15}$, dit-il. Il ressent avec désarroi la fin d'une époque artistique qui l'a formé. Goethe était encore attaché à trouver un équilibre entre art et vie. Le regard de Pfitzner sur le monde extérieur dans Palestrina est par contre beaucoup plus implacable que celui de ses prédécesseurs, Goethe, Grillparzer ou Wagner. Le compositeur allemand s'est lui-même positionné face au genre du Künstlerdrama en insistant sur l'idée artistique et philosophique située au centre de 
l'opéra, la séparation inexorable entre art et monde extérieur, telle que la concevait Schopenhauer :

Mais je ne voulais pas publier une histoire de la musique en vers et sous forme de scènes. La légende du sauvetage de la musique comme elle s'est formée me semble contenir une idée centrale incroyablement dramatique et de plus une idée philosophique et artistique, comme elle n'avait jamais été exprimée dans aucun drame d'artiste. La véritable grande œuvre d'art ne naît pas sous l'impulsion du monde, selon ses désirs ou sur ses ordres, mais au-delà du monde, elle sort de profondeurs métaphysiques ${ }^{16}$.

6 Sans être du Schopenhauer "versifié $»^{17}$, Palestrina est, après les œuvres de Wagner, la seule œuvre se fondant massivement sur la philosophie schopenhauerienne. La réception de Schopenhauer apparait chez Pfitzner comme considérable, sans commune mesure avec d'autres compositeurs de cette même époque tels que Humperdinck, Mahler, Schönberg ou Webern ${ }^{18}$. Schopenhauer est un philosophe que Pfitzner a lu avec une grande assiduité :

Il est le seul philosophe que j'aie lu entièrement et continuellement, et qui m'a donné beaucoup de jouissance, de connaissances, oui, a élevé mon âme et m'a consolé; et j'affirme que celui qui l'a vraiment compris ne peut plus s'en défaire ${ }^{19}$.

C'est en voulant illustrer l'idée de Schopenhauer que Pfitzner construit la structure de l'œuvre Palestrina, son triptyque, le contraste entre le monde de Palestrina fait d'introversion et celui du Concile, lieu d'agitation et de querelles où l'œuvre d'art devient «l'objet d'un marché politique »"

8 Avec Schopenhauer apparaît un des grands inspirateurs de la fin-de-siècle, un mouvement auquel se rattache le personnage de Palestrina par de nombreux côtés, même si l'opéra se situe dans une époque reculée, le $\mathrm{xvI}^{\mathrm{e}}$ siècle. Il n'est pas étonnant alors de constater l'identification profonde que connait Thomas Mann à la lecture de Palestrina, lui qui compte Schopenhauer parmi ses plus grands maîtres à penser. Palestrina est devenu sa "propriété », son «bien familier » ${ }^{21}$. Thomas Mann voit en Palestrina un artiste proche de ses propres aspirations et de celles de certains de ses contemporains, défenseurs de la Kultur, tandis qu'il voit en Silla, l'élève de Palestrina et l'admirateur du nouveau, une sorte de 'littérateur de la civilisation', obsédé par le progrès : il reconnaît en Palestrina un artiste dans une crise sans précédent car il est confronté à un monde en mutation, à des changements qu'il perçoit mais dont il se méfie, un témoin du finis musicae.

Pfitzner voit en effet dans l'évocation du "Sauveur de la Musique ", ainsi qu'on a appelé Palestrina depuis le XVII siècle, l'opportunité de dépeindre un artiste à la charnière de deux époques foncièrement différentes. Palestrina est le témoin même d'une époque finissante, a mûri dans l'atmosphère d'un temps révolu et soudain en voit poindre un autre auquel il appartient également par une infime partie de son être: « avoir un pied mettons dans le Moyen Âge et l'autre dans la Renaissance - ce n'est pas là une mince affaire $"^{22}$, pense Thomas Mann. Pfitzner connaît un même dilemme. Il écrit l'opéra Palestrina à une période marquée par des changements esthétiques, sociaux et politiques conséquents qu'il perçoit en restant toutefois prudent face à l'évolution du monde, son regard rivé sur le passé. Il se comprend lui-même comme un héritier de compositeurs tels que Brahms et Wagner, comme le dernier d'une longue tradition qu'il voit menacée, lui que l'on a appelé le «dernier romantique $»^{23}$ - et son œuvre Palestrina en porte les empreintes. 
10 Cet attachement au passé détermine en effet le personnage de Palestrina. Le premier acte s'ouvre sur l'opposition entre fidélité à la tradition musicale et modernisme résolu. Silla, le jeune élève de Palestrina, " adepte des futuristes florentins ${ }^{24}$, est porté par le désir de quitter Rome et son maître Palestrina, pour étudier une nouvelle forme de musique à Florence auprès de Bardi. Par opposition au besoin d'indépendance de Silla, Ighino, le fils de Palestrina et son porte-parole dans cette scène qui l'oppose à Silla, "délaisse volontiers l'éclat de la nouveauté et reste tourné vers l'ancien " ${ }^{25}$, prône les vertus de la tradition, le sentiment d'être «la petite partie d'un tout ${ }^{26}$. Palestrina a inculqué à ses disciples qu'il fallait être un maillon de la chaîne, s'appuyer sur une tradition séculaire pour créer, tandis que Silla, ne ressentant plus aucune dévotion pour les artistes l'ayant précédé, est attiré par une musique profane, nouvelle, inspirée "d'écrits païens, antiques ${ }^{27}$, croit que la musique religieuse polyphonique que représente Palestrina est «une vieille marchandise périmée» ${ }^{28}$. Palestrina n'est cependant pas aveuglé par son attachement au passé ; il a conscience que la musique est à un tournant ou arrive peut-être même à une fin. Plein de compréhension, il voit certes, avec le recul de l'expérience, les dangers d'un attachement trop exclusif à cette nouvelle musique, mais il ne peut en vouloir à Silla : " Je ne ressens en moi aucun droit de le lui interdire ${ }^{29}$ dit-il au cardinal Borromée. Palestrina n'est pas un conservateur borné comme Borromée, il sait que le monde est en train de changer, même si ce constat le pousse à la mélancolie :

Peut-être bien a-t-il raison! Qui peut savoir

Si le monde ne prend pas des chemins insoupçonnés

Et si ce qui nous semblait éternel, ne sera pas

Emporté par le vent ? ${ }^{30}$

11 Le cardinal Borromée, qui vient en émissaire du Pape lui demander d'écrire une messe qui tiendrait compte des exigences de Pie IV et sauverait l'art polyphonique de la destruction, s'offusque par contre du fait que Palestrina accepte chez lui, comme une fatalité, la nouvelle musique de Silla et lui rappelle que la tradition et l'Église sont semblables à un roc sur lequel est ancré l'art de Palestrina.

Cet attachement à la musique passée est rendu présent sur scène, dans le premier acte, par l'apparition des Maîtres de Palestrina qui, de l'au-delà, vont l'exhorter à la création et ont la réponse à sa question désemparée: "Pourquoi créer, se réjouir, souffrir, vivre ? ${ }^{31}$. De tout temps, ils ont été ses guides artistiques, ses accompagnateurs, ses plus fidèles alliés. Leur apparition vient rappeler à Palestrina cette filiation, une filiation qui défie la mort et le pousse à créer une dernière œuvre, à disposer « la dernière pierre de l'édifice $»^{32}$. Palestrina se voyant à la fin d'une grande époque - «Je suis un vieil homme mortellement las, au terme d'une grande époque $»^{33}$ - héritier de la tradition de la polyphonie, se comprend comme le dernier gardien de cet héritage :

Il [Palestrina] a l'impression que les mains de ses précurseurs se tendent vers lui, hors de leur tombe, eux qui ont vécu, créé, se sont battus et ont fait de grandes choses comme lui, et qu'ils l'invoquent : Toi qui est pareil à notre esprit, sauve nos œuvres. Et voilà qu'à présent sa vie, son travail assidu et calme ont un sens: il est prêt. $^{34}$

13 Dans ses propos sur Palestrina, Thomas Mann fait à juste titre référence à la polémique entre Pfitzner et Busoni à l'occasion du Projet d'une nouvelle esthétique de l'art musical de Busoni, «le livre-programme du progrès musical $»^{35}$ qui rappelle les positions de Palestrina et de Silla, l'opposition entre ancien et moderne. Pfitzner y répond par sa brochure au titre évocateur de Danger futuriste (1917). L'ouvrage de Ferruccio Busoni est 
le résultat d'une lassitude face aux œuvres musicales existantes. Busoni défend une démarche futuriste pendant que Pfitzner exprime son scepticisme devant un trop grand attachement à la modernité. Pfitzner retrace dans Danger futuriste l'attitude progressiste de Busoni et les dangers qu'elle cache à ses yeux :

De l'avenir, Busoni espère tout pour la musique occidentale, et il considère le présent et le passé comme un début balbutiant, une préparation. Mais s'il en allait autrement? Si nous nous trouvions sur un sommet, ou même si nous l'avions déjà passé ? Si notre siècle dernier, ou nos derniers cent cinquante ans, étaient l'apogée de la musique occidentale, le summum, la période véritablement éclatante, qui ne reviendra jamais et que suivra un déclin, une décadence comme après l'apogée de la tragédie grecque? Mon sentiment incline plutôt vers ce dernier point de vue. Déjà Rubinstein a parlé sérieusement d'un finis musicae. Qui sait si la mission de notre temps, au lieu de chercher des sixièmes de ton, de vouloir se ruer en avant à un rythme frénétique, de vouloir anéantir tout bien déjà acquis, pour l'amour d'une nouveauté - qui sait si la mission de notre temps ne serait pas plutôt de montrer que serait souhaitable une réflexion pleine d'amour sur ce qui fut et ce qui est conçu présentement et non uniquement sur ce qui flotte à la surface ? [...] L'époque précédente demandait, devant toute nouveauté: 'Cela m'est-il agréable et compréhensible?'. L'époque actuelle demande : 'Ne vais-je pas me déconsidérer, en faisant figure rétrograde ?' Toute la différence est là . ${ }^{36}$

Pfitzner voit la musique passée comme le fondement de toute musique future et refuse de faire table rase. À ses yeux, une évolution vieille de plusieurs centaines d'années ne peut pas être balayée pour les besoins d'une vision futuriste trop incertaine. Lorsque Busoni écrit que l'on reconnaît l'énergie créatrice au fait qu'elle s'émancipe des traditions $s^{37}$, Pfitzner ne peut lui accorder son consentement. Pfitzner met en garde contre la croyance au progrès comme une évolution vers plus de qualité. Il s'oppose à l'idolâtrie exclusive du nouveau qui met à mal toute forme d'art le précédant. Pour lui, l'exigence de progrès seule est insuffisante pour donner des fondations viables et saines à une culture. Pour autant, Pfitzner ne refuse pas toute forme de progrès. Bernhard Adamy a raison de dire dans son ouvrage sur Hans Pfitzner qu'il faut éviter le qualificatif de réactionnaire pour comprendre le compositeur. Pour Pfitzner, la tradition doit déterminer "comme un sentiment vivant, de l'intérieur, les lois de l'évolution du monde $»^{38}$, ne doit pas être une contrainte pour le créateur, mais un enrichissement. Il ne faut ainsi pas en conclure que Pfitzner est globalement réfractaire à son époque. Comme le montre Adamy, Pfitzner a aussi contribué à promouvoir la musique de son temps ${ }^{39}$, même si l'avenir de la musique lui apparaît comme incertain.

Une réconciliation entre tradition et modernité n'est pas envisagée dans Palestrina, même si Palestrina accepte, plus par lassitude et grandeur d'âme que par conviction, le départ de Silla pour Florence. Dans une conversation que Pfitzner a avec Thomas Mann pendant la Première Guerre mondiale, le compositeur insiste sur la différence sans équivoque, dans les derniers tableaux scéniques, entre les Maîtres chanteurs de Wagner et Palestrina. Chez Wagner, il voit "une scène rayonnante de lumière: allégresse populaire, fiançailles, éclat et gloire ». Dans Palestrina par contre, à la fin du dernier acte, ne reste sur scène qu'un compositeur « fêté il est vrai, lui aussi », mais « tout seul dans la pénombre de sa chambre $»^{40}$. Pfitzner en conclut : «Les Maitres sont l'apothéose du nouveau, un hymne en l'honneur de l'avenir et de la vie. Dans Palestrina, tout incline vers le passé. Il y règne une sympathie pour la mort $»^{41}$.

Pfitzner nous dit s'être penché sur la vie de Palestrina, car il a été touché par l'artiste du XVI ${ }^{e}$ siècle qui n'a jamais quitté Rome, a vu les Papes se succéder et n'a fait toute sa 
vie que se consacrer à sa tâche silencieuse avec exclusivité et dévouement, "vivant paisiblement dans l'ombre $\aleph^{42}$. Il admire en Palestrina le fait d'avoir perpétué la tradition en ayant écrit une messe «dont le style ne désavouait pas les conquêtes du grand art des derniers siècles ${ }^{43}$, mais aussi d'avoir rendu l'accès des chants religieux à l'homme simple en ayant enrayé les excès de la polyphonie, d'avoir ainsi rempli l'exigence d'une réforme de la musique. L'acte III se termine ainsi sur un Palestrina plongé dans l'obscurité du jour finissant, ayant accompli sa tâche. Il est calme, perdu dans ses pensées musicales, sur le visage l'expression d'une grande dévotion. Sous ses fenêtres, les « Evviva Palestrina » du 'monde' ne peuvent atteindre le maître, assis à son orgue. Le dernier acte de Palestrina se termine certes sur la consécration du maître, mais la scène finale rend perceptibles les lois toutes particulières de la création et ne peut effacer le désaccord profond qui règne entre art et monde extérieur dans Palestrina.

\section{NOTES}

1. «Gipfel im musikalischen Schaffen unserer Epoche» (Palestrina. Programmheft zur Neuinszenierung. Bayerische Staatsoper, München, 1979, p. 75).

2. «Ohne daß ich etwa andere Bühnenwerke von mir, wie ,Das Herz', die ,Rose vom Liebesgarten musikalisch hinter den ,Palestrina' stelle, so muß ich ihn doch als mein Hauptwerk, sozusagen das Werk meines Lebens betrachten, da er das einzige Bühnenwerk ist, von dem auch die Dichtung ganz und gar von mir stammt, sowohl in der Konzeption als in der Ausführung» (H. Pfitzner: Mein «Hauptwerk», p. 40).

3. Lettre au Théâtre National de Munich, 20.2.1929 (in: B. Adamy: Das Palestrina-Textbuch als Dichtung, in: Symposium Hans Pfitzner Berlin 1981, Tutzing, Hans Schneider, 1984, pp. 21-22).

4. «Mit ihrem lauten und wilden Getriebe, die sich in der Zeitlichkeit abrollt» (H. Pfitzner : Palestrina. Ein Vortrag über das Werk und seine Geschichte, in: H.P: Sämtliche Schriften, herausgegeben von Bernhard Adamy, t. 4, Tutzing, Hans Schneider, p. 422).

5. «Denn ich fühlte mich nicht als Dichter und hatte ja auch nie mich auf diesem Gebiet versucht [...]» (H. Pfitzner: Palestrina. Ein Vortrag über das Werk und seine Geschichte, op. cit., p. 418).

6. « Ich erinnere mich, daß ich mich in dieser ganzen Zeit gar nicht als Komponist fühlte » (Ibid., p. 425).

7. «Du kennst wohl meine Ansicht über Opern-Dichtung und Dichten überhaupt; und weißt, daß sie keine frivole ist, und wie ich Leute verachte, die ohne Dichter zu sein, sich daran machen, sich selber 'Texte' zu schreiben, die sie für Dichtungen halten» (lettre du 14.11.1909, cité d'après B. Adamy: DasPalestrina-Textbuch als Dichtung, op. cit., p. 22).

8. Th. Mann: Considérations d'un apolitique. Traduit de l'allemand par Louise Servicen et Jeanne Naujac, Paris, Grasset, 1975, p. 344.

9. «Der Plan, den großen römischen Kirchen-Komponisten zum Helden eines Dramas zu machen, reicht in sehr frühe Zeiten meines Lebens. Er kam mir schon, als ich in Mainz ein kleiner Kapellmeister am Stadttheater war, in den neunziger Jahren des vorigen Jahrhunderts» (Palestrina. Programmheft zur Neuinszenierung, op. cit., p. 40).

10. «Die erste und stärkste Anregung zu der Konzeption meines Werkes» (H. Pfitzner: Palestrina. Ein Vortrag über das Werk und seine Geschichte, op. cit., p. 419). 
11. «Über dem weltlichen Treiben, dem eigentlich realen, vom Willen geführten Leben der Völker», «neben der Weltgeschichte geht schuldlos und nicht blutbefleckt die Geschichte der Philosophie, der Wissenschaften und der Künste» (A. Schopenhauer: Parerga und Paralipomena, § 52).

12. B. Adamy: Das Palestrina-Textbuch als Dichtung, op. cit., p. 53.

13. «Auch ,Palestrina' von Hans Pfitzner (1917) steht in der Gattungstradition des historisierenden Komponisten-Portraits, die bereits wenig später - mit Paul Graeners „Friedemann Bach‘ (1931) - ausklingen sollte» (S. Kohler: Der Komponist als Opernheld. Zur Gattungstradition von Pfitzners ,Palestrina', in: Palestrina. Programmheft zur Neuinszenierung, op. cit., p. 37).

14. Cf. R. Seebohm: Triumph und Tragik des Künstlertums. Die Stellung von Pfitzners, Palestrina in der Geschichte des deutschen Künstlerdramas, in: Mitteilungen der Hans Pfitzner Gesellschaft, avril 1974, p. 10-35.

15. «Unser Leben wird immer maschineller, immer nüchterner, aller Innerlichkeit abholder» (H. Pfitzner: WebersFreischütz, in: H.P: Gesammelte Schriften, Augsburg, 1926, t. 1, p. 79).

16. «Aber ich wollte ja keine Musikgeschichte in Verse und Szenen bringen. Die Legende von der Rettung der Musik, wie sie sich nun einmal herausgebildet hatte, schien mir einen ungeheuer dramatischen Kern zu enthalten und überdies eine kunstphilosophische Idee, wie sie noch in keinem Künstler-Drama ausgesprochen war. Das echte große Kunstwerk entsteht nicht mit der Welt, auf deren Wunsch oder Befehl [...] sondern über die Welt hinweg, es taucht aus metaphysischen Tiefen auf» (H. Pfitzner: Mein «Hauptwerk», ibid., p. 41).

17. «Es wäre falsch, in ,Palestrina' gereimten Schopenhauer zu sehen» (B. Adamy: Hans Pfitzner. Literatur, Philosophie und Zeitgeschehen in seinem Weltbild und Werk, Tutzing, Hans Schneider, 1980, p. 161).

18. Cf. ibid., p. 144.

19. «Er ist der einzige Philosoph, den ich vollständig und immer wieder gelesen habe, und der mir großen Genuß, Belehrung, ja, zu Zeiten Erhebung und Trost gewährt hat; und ich behaupte, daß, wer ihn wirklich verstanden hat, nie wieder von ihm loskommen kann» (H. Pfitzner: Reden, Schriften, Briefe. Herausgegeben von Walter Abendroth, Berlin, 1955, p. 35).

20. Th. Mann: Considérations d'un apolitique, op. cit. , p. 345.

21. Ibid., p. 341.

22. Ibid., p. 351.

23. Notion véhiculée notamment par Conrad Wandrey. À tort pour beaucoup d'interprètes.

24. Th. Mann: Considérations d'un apolitique, op. cit., p. 343.

25. Ibid., p. 342.

26. «Als kleines Stück / Von einem Ganzen» (I, 2).

27. «Heidnischen, antiken Schriften» $(\mathrm{I}, 3)$.

28. «Abgegriff'ne alte Ware» (I, 3)

29. «Zu wehren ihm fühl' ich in mir kein Recht» $(\mathrm{I}, 3)$.

30. «Vielleicht wohl hat er recht! Wer kann es wissen, / ob jetzt die Welt nicht ungeahnte Wege geht, / Und was uns ewig schien, nicht wie / Im Wind verweht?»

$(\mathrm{I}, 3)$.

31. «Wozu das ganze Schaffen, Freuen, Leiden, Leben?» (I, 4).

32. «Den Schlußtein zum Gebäue» $(I, 5)$.

33. «Ich bin ein alter, todesmüder Mann / Am Ende einer großen Zeit» (I, 5)

34. «Es ist ihm, als streckten sich die Hände seiner Vorgänger, die gleich ihm gelebt, geschaffen, gerungen und gewirkt haben, aus dem Grabe und riefen ihn an: Du, der Du von unserem Geiste bist, rette unsere Werke! Und siehe da, nun lohnt sich sein Leben, seine stete Arbeit in der Stille : er ist bereit!» (H. Pfitzner: Palestrina. Ein Vortrag über das Werk und seine Geschichte, op. cit., p. 421).

35. Th. Mann: Considérations d'un apolitique, op. cit., p. 347. 
36. Le passage de l'ouvrage de Busoni est traduit en français dans ibid., p. 347-348.

37. Cf. B. Adamy : Hans Pfitzner, op. cit., p. 92.

38. Ibid., p. 94.

39. Cf. ibid., p. 100.

40. Th. Mann: Considérations d'un apolitique, op. cit., p. 354.

41. Ibid. On a beaucoup disserté sur le terme de "sympathie pour la mort». C'est par lui que Thomas Mann, qui lui-même avait appliqué ce terme à son roman La Montagne magique, a vu, à juste titre, en Pfitzner un héritier du romantisme tardif inspiré de Schopenhauer et de Wagner et qu'il a pensé que le génie inspirateur de Pfitzner n'était pas la vie mais la mort.

42. «Still im Dunkel lebend» (H. Pfitzner: Mein «Hauptwerk», in: Palestrina. Programmheft zur Neuinszenierung, op. cit. p. 40).

43. «Deren Stil die Errungenschaften der großen Kunst in den letzten Jahrhunderten nicht verleugnete» (Ibid.)

\section{RÉSUMÉS}

La légende en trois actes, Palestrina, de Hans Pfitzner est, comme le remarque Thomas Mann à juste titre dans ses Considérations d'un apolitique, « le dernier écho et, consciemment, l'ultime de la sphère schopenhauerienne-wagnérienne ». Pfitzner se définit lui-même comme un héritier d'une longue tradition qu'il voit menacée par les bouleversements artistiques et politiques que connaît le début $\mathrm{du} \mathrm{xx}^{\mathrm{e}}$ siècle et il déplore, comme Thomas Mann dans son œuvre conservatrice, le finis musicae ; ceci notamment dans sa polémique contre Ferruccio Busoni au titre évocateur de Danger futuriste (1917) dans laquelle on retrouve certaines positions de l'opéra Palestrina.

Palestrina fait partie du genre littéraire du Künstlerdrama dont Pfitzner reprend le thème principal depuis Goethe, l'opposition entre art et vie, en s'appuyant sur la philosophie schopenhauerienne. Contrairement à ses prédécesseurs, Pfitzner décrit dans Palestrina l'antagonisme entre art et vie avec une dureté implacable.

Palestrina, le musicien $\mathrm{du} \mathrm{XvI}^{\mathrm{e}}$ siècle que le siècle suivant a surnommé le "Sauveur de la Musique", s'avère être un personnage typique d'une "situation culturelle tardive" (R. Seebohm). Sa réponse aux bouleversements de son temps s'exprime par un attachement sans faille aux grands compositeurs du passé, sachant qu'il se situe lui-même «à la fin d'une grande époque ».

Pfitzners Legende in drei Akten, Palestrina, ist, wie Thomas Mann in seinen Betrachtungen eines Unpolitischen zu Recht bemerkt, «etwas Letztes und mit Bewußtsein Letztes aus der schopenhauerisch-wagnerischen» Sphäre. Pfitzner definiert sich selbst als Spätgeborener und Erbe einer jahrhundertelangen Tradition, die er durch die künstlerischen Umwälzungen am Anfang des 20. Jahrhunderts gefährdet sieht und er beklagt, wie Thomas Mann auch in seiner konservativen Schrift, das finis musicae, so u. a. in seiner Polemik gegen Ferruccio Busoni Futuristengefahr (1917), in der wir Positionen wiederfinden, die er in seinem Palestrina eingenommen hat. Geistesgeschichtlich gehört Palestrina zu der Gattung des Künstlerdramas, dessen Hauptthema, den Widerspruch zwischen Kunst und Leben, Pfitzner in der Nachfolge Schopenhauers aufgreift. Im Verhältnis zu seinen Vorgängern behandelt jedoch dieses späte Künstlerdrama den Kunst-und-Leben-Antagonismus mit unversöhnlicher Härte. 
Palestrina, die Musikergestalt aus dem 16. Jahrhundert, den man seit dem 17. Jahrhundert den «Retter der Musik» genannt hat, erweist sich als eine typische Figur einer Übergangzeit und einer «Spätkultursituation» (R. Seebohm). Seine Antwort auf die Umwälzungen seiner Zeit ist eine Rückwärtsgewandtheit, die ihn jedoch nicht daran hindert, im Bewußtsein ein Letzter zu sein und am «Ende einer grossen Zeit» zu stehen, Großes zu leisten.

INDEX

oeuvrecitee Palestrina, Danger futuriste

Mots-clés : Künstlerdrama

\section{AUTEURS}

\section{JOËLLE STOUPY}

Université du Littoral-Côte d'Opale (Boulogne-sur-Mer) 